\title{
Disminución en la frecuencia de uso en maíces de color en dos regiones de Tlaxcala
}

\author{
Decrease in the frecuency of use of colored corn in two regions of tlaxcala \\ Orozco-Bolaños Hermila ${ }^{1 凶}$, García-Juárez Guillermina ${ }^{1}$, Hernández-Vázquez Maricela ${ }^{1}$ y Juárez-Ortiz María \\ Guadalupe $^{1}$ \\ 1Universidad Autónoma de Tlaxcala. Facultad de Agrobiología. Km 10.5 Autopista Tlaxcala-San Martín \\ Texmelucan, Ixtacuixtla, Tlax. C.P. 90120 . Tel: 012484815382.
}

${ }^{\square}$ Autor para correspondencia: emy_r1@yahoo.com

Recibido: 17/01/2016
Aceptado: 20/06/2016

\section{RESUMEN}

La agrodiversidad es un componente de los sistemas de producción agrícola tradicional, una de las características es el uso de semillas de maíz criollo de diversos colores. En este estudio se analizó la disminución en la frecuencia de uso en maíces de color en dos regiones del estado de Tlaxcala. La población de productores de maíz se obtuvo del programa de apoyos directos al campo, se seleccionaron 5 localidades en cada región con características similares entre ellas, pero contrastantes entre regiones. Se encuestó a 138 productores, analizando la información mediante estadística descriptiva e inferencial. Los resultados obtenidos muestran la disminución en el uso de maíces de color en la siembra, principalmente en la región poniente, también que el productor los está abandonando por la falta de mercado, apoyo económico y la migración, situaciones que amenazan la seguridad alimentaria en las regiones de estudio.

Palabras clave: Seguridad alimentaria, variedades, colores de maíz, usos, regiones

\begin{abstract}
ABSTRAC
Agrodiversity is a component of traditional agricultural production systems, one of the features is the use of seeds of landraces of different colors. In this study we analyzed the decline in the frequency of use in color corns in two regions of the state of Tlaxcala. The population of corn farmers was obtained from the Program of Direct Support to the Field, 5 locations in each region with similar characteristics were included, but contrast between regions was also selected. We surveyed 138 producers, analyzing the information using descriptive and inferential statistics. The results show a decrease in the use of corn color in planting, mainly in the western region, also the producer is abandoning by the lack of market, economic support and migration situations that threaten food security in study regions.
\end{abstract}

Keywords: food safety, variety, colored corn, use, regions. 


\section{INTRODUCCIÓN}

La agricultura tiene alternativas para la obtención de alimentos, específicamente la agricultura campesina, la cual se caracteriza por sembrar cultivos tradicionales (Hernández, 1985 y Bartra, 1995), la producción de maíz es uno de ellos, su particularidad es el uso de diferentes variedades criollas de semilla mismas que se identifican por sus colores; blanco, amarillo, azul, rojo, rosa, negro, púrpura, crema, entre otros. A nivel mundial la producción de maíz se ubica en primer término (Muñoz, 2003), superando al trigo y al arroz; es el de mayor diversidad no sólo genética sino también de uso. Su origen, de acuerdo a Massieu y Lechuga (2002), Muñoz (2003), Escobar (2006) y Gil y Álvarez (2007) es México y Mesoamérica, en nuestro país se han reportado entre 40 a 59 razas (Reyes et al. 2005, Gil y Álvarez, 2007, Fitting, 2007, Madrigal, 2008). Hay diversos estudios en México relacionados al maíz criollo de colores como azul, rojo, púrpura, blanco, amarillo; algunos de ellos reportan las características agronómicas, calidad del grano y tortilla, la nixtamalización, las propiedades físicoquímicas, así como el contenido de carotenoides y antocianinas de importancia médica (María et al. 2003; Vázquez et al. 2010; Rangel-Meza et al. 2004; Salinas et al. 2008; Espinosa et al. 2010). Otros trabajos realizados en localidades de Tlaxcala han reportado de 12 a 15 tipos de variedades de maíz predominando el blanco, azul, rojo y cacahuazintle, además de moradillo, palomero, cónico, ancho, chalqueño, pepitilla chica, blanco cremoso, blanco grande, amarillo, cremoso chico, cañuela y chalco chico; (Lara et al. 2002; Muñoz, 2003; Reyes et al. 2005; Madrigal, 2008 y María y Hernández, 2010). Lo anterior refiere riqueza genética de maíces para el estado los cuales están relacionados con la selección, intercambio y conservación que realiza el productor, prácticas agrícolas que pertenecen a la agricultura de temporal, una característica de Tlaxcala es, del total de la superficie sembrada de maíz, el $82 \%$ es temporal y 18\% riego (SAGARPA, 2007), generalmente el sistema de producción es tradicional, misma que llevan a cabo productores de autoconsumo que de acuerdo a lo referido por Villa (2008) representan el 82\% del total nacional. Como se observa existe una relación agricultura tradicional y uso de variedades de maíces criollos, ésta práctica ha asegurado la producción que se ha mantenido por muchos años. La selección de semilla para la siembra es una práctica muy cuidadosa que realiza el productor en el momento de la cosecha, y se relaciona con seguridad alimentaria de las familias. Falta realizar más investigación para ampliar el conocimiento acerca de las variedades de maíz de color que existen en las regiones, asimismo no se ha realizado análisis como el color del maíz y su relación con seguridad alimentaria. El objetivo de esta investigación fue analizar la disminución en la frecuencia de uso en maíces de color en dos regiones de Tlaxcala.

\section{MATERIALES Y MÉTODOS}

Se seleccionaron las regiones de estudio con base en los criterios de mayor y menor superficie sembrada de maíz, así como las características geográficas, climáticas, sociales y tradicionales, la información se obtuvo del Instituto Nacional de Estadística Geografía e Informática del Anuario Estadístico (INEGI, 2007) y Gobierno del estado de Tlaxcala (1998). Las regiones fueron dos; la Oriente por presentar la mayor superficie sembrada de maíz, los municipios fueron Huamantla y Cuapiaxtla, y el Poniente con menor superficie, comprendió Ixtacuixtla y Españita ubicados al oeste del estado. La población de productores de maíz se obtuvo en el Centro de Apoyo al Desarrollo Rural (CADER) de Ixtacuixtla y Huamantla y de la Secretaria de Agricultura Ganadería Desarrollo Rural y Pesca y 
Alimentación (SAGARPA). Los datos seleccionados fueron: población total de productores por localidad y superficie sembrada de maíz de temporal. Con esta información se seleccionaron 5 localidades por región. La muestra fue de 138 productores en total, se visitaron las localidades en las que se aplicó el cuestionario, para estructurarlo se consideraron algunos aspectos de los trabajos de Escobar (2006) y Osorio-García et. al. (2012). El instrumento se conformó de 14 reactivos y de dos apartados: 1. Datos generales del productor, 2. Uso de la tierra y diversidad en el cultivo de maíz. Para la interpretación de la información se utilizó estadística descriptiva e inferencial de ésta última una prueba de $t$ student, Ji cuadrada y análisis de varianza.

\section{RESULTADOS}

En relación a las características sociodemográficas de los productores que atienden el cultivo de maíz en las dos regiones de estudio, se observó que el género masculino es quien principalmente atiende el cultivo, sin embargo, hay presencia de la mujer en este proceso, el $81 \%$ de los productores son mayores de cincuenta años; en escolaridad más del $50 \%$ cursó solo primaria, dedicándose desde sus primeros años de vida en la selección y mantenimiento de las semillas criollas, conocimiento que les fue transferido de padres

a hijos. Respecto al área sembrada y el rendimiento de maíz en las regiones analizadas, el promedio en predios va de 1.8 a 1.4 hectáreas, ligeramente mayor en la región Poniente, con mayor superficie fraccionada por sus características orográficas, así mismo la superficie sembrada es menor en el Poniente con 3.44 hectáreas, al Oriente es 5.43; en el rendimiento comparativamente no hubo diferencias significativas (2.04-2.43 ton/ha).

Correspondiente a las semillas de maíz que siembran los productores en las zonas de estudio, el análisis de contraste de hipótesis permitió evidenciar que la diversidad observada a partir de la denominación "color" expresada por los productores diferenció de manera cualitativa las preferencias del grano relacionadas con las características de cada región, por tanto, la diversidad es diferente en ambas regiones (Figura 1). Existe más variedad en la región Oriente ya que se cultivan cinco tipos de maíz criollo, que en la Poniente. Las proporciones señalan que la diversidad se está perdiendo al menos en cuanto a colores.

Figura 1. Colores de semillas de maíz en dos regiones



Con respecto al destino que le da el productor al maíz criollo, no existen diferencias significativas en el uso entre las dos regiones, se puede ver que cerca del $50 \%$ se destina al consumo familiar, al consumo animal se dispone del 19-22\%, para venta entre el 24$29 \%$ (Figura 2). Respecto a la conservación de la semilla in situ, se observa un 5 y $6 \%$. Los resultados nos indican que las preferencias en el uso del maíz, es el consumo humano, por sus 
Figura 2. Usos de la semilla de maíz criollo en dos regiones.



Específicamente el flujo de la semilla de maíz, esto es, como el productor obtiene los granos para sembrar en cada ciclo agrícola. En la región Oriente resultó de mayor importancia la selección y compra de la semilla, en el
Poniente predomina la compra y regalada por el padre (Figura 3). Este flujo de germoplasma tiene íntima relación con los usos, debido a que la alimentación depende de la conservación in situ del maíz en las localidades o zonas rurales.

Figura 3. Obtención de la semilla de maíz

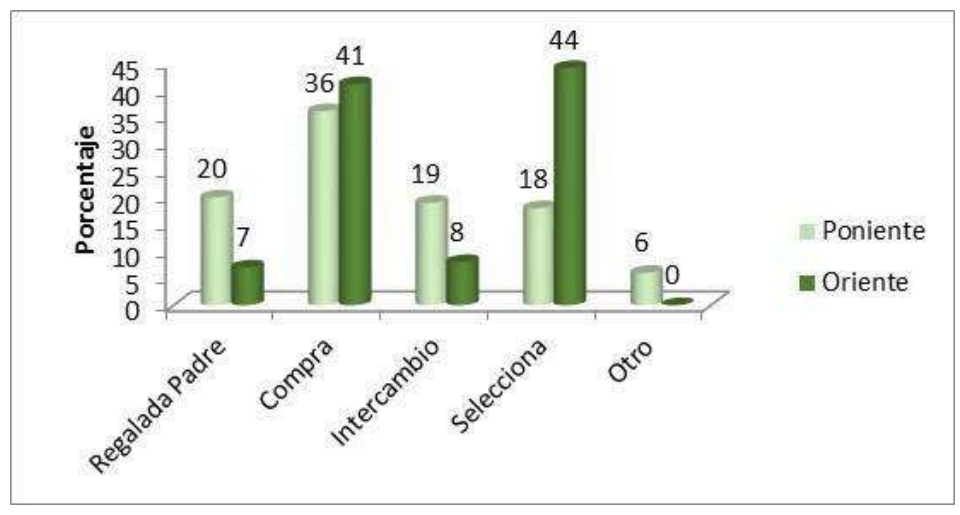

El Cuadro 1, muestra el maíz criollo en riesgo de perderse para ambas regiones, $67 \%$ de los productores así lo señaló, al compararlas se observa que en el Poniente los principales son cañuela $17 \%$, negro $15 \%$, azul, cacahuazintle y morado con $10 \%$ respectivamente. El maíz que se está abandonando en el Oriente es amarillo con $22 \%$, ancho y cacahuazintle $19 \%$ cada uno y arrocillo $14 \%$, aún y cuando es diferente el porcentaje, la tendencia es que cinco colores están en peligro. Este comportamiento se relaciona con las preferencias de la demanda del mercado. 
Cuadro 1. Maíz que se está abandonando en dos regiones del estado de Tlaxcala

\begin{tabular}{|c|c|c|}
\hline Forma o color & $\begin{array}{c}\text { Región Poniente } \\
\mathbf{\%}\end{array}$ & $\begin{array}{c}\text { Región Oriente } \\
\mathbf{\%}\end{array}$ \\
\hline Azul & 10 & 8 \\
\hline Amarillo & 10 & 22 \\
\hline Cañuela (blanco) & 17 & 0 \\
\hline Ancho (blanco) & 2 & 19 \\
\hline Cacahuazintle (blanco) & 10 & 19 \\
\hline Morado & 10 & 0 \\
\hline Pinto & 5 & 0 \\
\hline Blanco & 8 & 3 \\
\hline Palomero (amarillo) & 2 & 0 \\
\hline Rojo & 5 & 3 \\
\hline Rosa & 2 & 0 \\
\hline Pinolero (blanco) & 2 & 0 \\
\hline Cremoso (crema) & 2 & 6 \\
\hline Negro & 15 & 6 \\
\hline Arrocillo (blanco) & 0 & 14 \\
\hline
\end{tabular}

\section{DISCUSIÓN}

La percepción sobre la preservación de la diversidad del maíz, como parte de la seguridad alimentaria que representa para los campesinos del área de estudio, coincide con el concepto de Alarcón-Chaires, (2010) señalando que en la producción de alimentos agrícolas es importante mantener la diversidad de las semillas, como resultado de la relación cultural de los campesinos con la naturaleza, de ahí que la diversidad es un significado que deriva acciones y soluciones ante la crisis ambiental, alimentaria y social a través de un sistema cognitivo y de prácticas de manejo adecuadas para la conservación de los agroecosistemas como base productora de la diversidad alimentaria.

México es reconocido como el cuarto país más rico en diversidad biológica y como centro de origen de especies como el maíz, éste constituye la base de la alimentación de los mexicanos, y las expresiones culturales desarrolladas por las comunidades son consideradas de una riqueza social invaluable (Sarmiento y Castañeda, 2011), se reconocen entre 40 a 59 razas (Gil y Álvarez 2007, Fitting, 2007, Madrigal 2008, Reyes et al. 2005), en
Tlaxcala a pesar de ser un estado pequeño se han identificado entre 12 y 15 razas (María y Hernández, 2010) lo que representa el $20 \%$ a nivel nacional, por lo anterior el sistema de producción de maíz criollo de temporal en el estado es muy importante para la conservación del grano, los responsables de esta labor son los "productores de subsistencia-autoconsumo o campesinos", su propósito es asegurar la alimentación de la familia así como, garantizar la autosuficiencia y seguridad alimentaria (Castro, 2006 y Santos et al. 2014) sin embargo, las nuevas generaciones no se están incorporando en las labores del campo para la obtención de maíz, quienes atienden esta actividad son personas mayores de cincuenta años y con pocos años de escolaridad, esto coincide con la CEPAL (2014) al destacar que los jefes de hogares agrícolas-familiares tienen las mayores edades promedio y los menores niveles de educación. Esto lleva a la reflexión, la pérdida de maíces de colores está íntimamente relacionada con los saberes culturales milenarios, esto es, una simbiosis entre hombre y semilla porque el maíz necesita de la intervención del ser humano para sobrevivir, desde su domesticación hasta la adaptación de miles de variedades de semillas nativas que son producidas, mejoradas, salvaguardadas y adaptadas a los diferentes climas y condiciones edáficas (Madrigal, 2008 
y Álvarez-Buylla et al. 2011), si ya no hay nuevas generaciones que les interese trabajar el cultivo entonces, los conocimientos ancestrales y tradicionales del maíz, corren el riesgo de perderse amenazando la seguridad alimentaria.

Los conocimientos ancestrales que posee el productor y que definen su relación con el medio físico y los elementos bióticos

(Castro, 2006), permiten que independientemente de los contrastes orográficos, climáticos y edafológicos así como, la diversidad de características espaciales y temporales (Toledo, 1994 citado por Núñez et al. 2003 y López et al. 2007), que presentan los diversos agro-ecosistemas, se fortalezcan con el valor que los campesinos le dan al maíz, de ahí la importancia para preservar sus variedades a través de la selección de semilla de mayor

rendimiento (tonelada por hectárea), considerando lo anterior se encontró que no hubo diferencias entre el promedio de las regiones, esto es 2.04 y 2.43 toneladas por hectárea a pesar de que el relieve y la superficie sembrada son totalmente diferentes. Entonces, se puede decir que estos rendimientos dependen de las prácticas de cultivo que realizan los productores en cada región, esto es, el conocimiento tradicional que permite que ellos busquen la forma de mantener su producción; ejemplo, al Poniente los agricultores seleccionan y conservan las mejores mazorcas (más grandes, mejor color, carretes llenos, no presenten plagas o enfermedades), al Oriente la costumbre en algunos casos es cambiar la semilla cada dos o tres años con otros productores o localidades cercanas, buscan las mejores y con ello evitar el agotamiento del suelo, estas son prácticas agroecológicas que junto con las características de los maíces criollos; ser resistentes a plagas, requerir menos agroquímicos, poseer mejor capacidad de adaptación tanto a sequías, suelos pobres y condiciones climáticas extremas (Perales et al. 2003, Soleri et al. 2006, Hernández et al. 2015), se demuestra que los productores de cualquier región tienen en común los conocimientos ancestrales que les permiten obtener un rendimiento adecuado.

Se ha mencionado que en Tlaxcala existe una gran riqueza del maíz en sus diferentes colores y formas, sin embargo el productor los está abandonando, otra de las causas es la falta de mercado, Escobar (2006); Pérez et al. (2006) y Vázquez et al. (2010) señalan que el maíz blanco tiene mejor precio y los industriales de la masa y la tortilla prefieren procesar blancos y/o crema, que maíces de otros colores, también se enfrentan a la introducción de semillas de maíz mejorado, migración, falta de apoyo económico, marginación y pobreza, otra amenaza es el clima poniendo en riesgo las razas nativas (Bartra, 1995; Hernández et al. 2015), son motivos suficientes para el abandono de maíces criollos y, en estas dos regiones solo son cuatro los que se siembran de manera intensiva; el blanco, amarillo, azul y crema de quince tipos de granos entre colores y tamaños que reportan, tales como: blanco (cañuela, ancho, palomero,

pinolero, arrocillo, (cacahuazintle), azul, amarillo, morado, pinto, rojo, rosa, crema y negro; al menos en cuanto a colores y proporciones este escenario nos muestra el riesgo de perder biodiversidad.

Para el productor lo más importante es alimentar a su familia de manera segura y sana, debido a que en las regiones de estudio, se destina el 46 y $47 \%$ de su producción de maíz al consumo humano, esta situación coincide con Gil y Álvarez (2007), al señalar que el 57\% de la producción nacional de maíz se destina a la alimentación, por ello México es rico en platillos tradicionales a base de éste grano (Smale y Bellon, 2001), para elaborar tortillas, atole, esquites, elotes, gorditas, tlacoyos, tlaxcales, quesadillas, tamales chileatole, memelas, pinole (Gil y Álvarez, 2007 y Meléndez, 2015), desde el punto de vista nutrimental, se estima que el consumo por persona es de $300 \mathrm{~g} / \mathrm{día}$, aporta el $56 \%$ de las calorías y el 47\% de proteína (González, 1995 
y Massieu y Lechuga, 2002), los granos contienen entre 4 y $7 \%$ de lípidos, 8 a $11 \%$ de proteína y 69 a $86 \%$ de carbohidratos (MéndezMontealvo et al. 2005, Castañeda-Sánchez, 2011, Salinas et al. 2013), además de vitamina A y E, algunos aminoácidos esenciales fenilalanina, histidina, isoleucina, leucina, lisina, metionina, treonina, triptófano y valina (Castañeda-Sánchez, 2011) esto es seguridad alimentaria, al acceder a alimentos seguros y nutritivos (FAO, 2016). En la parte médica se ha encontrado que las semillas de colores azules, rojos, negros, morados y púrpuras contienen antocianinas importantes en la

prevención de enfermedades humanas (Espinosa et al. 2010 y Aguilera et al. 2011), son compuestos antioxidantes los cuales retrasan el daño causado por los radicales

libres, con actividad anticancerígena, antiinflamatorio, antidiabético, antitumorales, además mejora la agudeza visual y del comportamiento cognitivo (Castañeda-Sánchez, 2011 y Aguilera et al. 2011). En las regiones de estudio se encontró que no existen diferencias significativas en el destino que le dan los agricultores a su producción, esto tiene sentido porque en ambas regiones son productores que cultivan para el autoconsumo, otra parte la destinan como forraje y venta. Algo que llama la atención es la práctica de selección y conservación in situ del maíz, al guardar en cada ciclo agrícola entre el 5 y $6 \%$ de su producción para asegurar el siguiente ciclo mediante su banco de germoplasma. Finalmente se destaca la necesidad de potenciar la demanda de maíces nativos a partir de la revalorización de los usos tradicionales (Fernández et al. 2013) que benefician a la sociedad.

\section{CONCLUSIÓN}

Es inminente la pérdida del germoplasma tan colorido de maíz en estas regiones de Tlaxcala por diversas causas, como la edad de los productores que rebasan los 50 años, lo que plantea un reto generacional. Otro aspecto es la falta de mercado para la comercialización de las semillas, por falta de conocimiento del uso del maíz, las costumbres van cambiando y es importante que los saberes ancestrales y tradicionales de su uso continúen, ya que corremos el riesgo de perder diversidad y cultura, regalos para la humanidad. Los diferentes usos expresan diversidad y seguridad alimentaria, entonces desde lo social y cultural se debe revalorizar en el núcleo familiar y proyectarlo hacia la sociedad por los beneficios nutricionales y medicinales que aporta. Es importante proponer políticas públicas para la preservación de la diversidad del maíz, promoviendo su siembra y consumo, dada la importancia que tienen sus propiedades al consumirlo y las características agronómicas para su cultivo, el conocimiento autóctono que tiene el productor es trascendental que no se pierda, ya que esto impactará directamente en nuestra seguridad alimentaria.

\section{LITERATURA CITADA}

Alarcón-Chaires, P. 2010. Etnoecología de los indígenas $\mathrm{P}$ 'urhèpecha. Una guía para la aproximación de la apropiación de la naturaleza. UNAM, México.

Álvarez-Buylla, R.E; Carreón, G.A y San Vicente, T.A 2011. Haciendo milpa. La protección de las semillas y la agricultura campesina. UNAM, México.

Aguilera, O. M; Reza V.M.D.C; Chew, M.R.G y Meza, V.J.A. 2011. Propiedades funcionales de las antocianinas. Revista biotecnia XIII (2): $16-22$ https://doi.org/10.47808/revistabioagro.v4i1.2

Bartra, A. 1995. Los nuevos campesinos En JeanFrancois Prud’homme (coordinador). El impacto social de las políticas de ajuste en el campo mexicano. Ed Plaza y Valdés, México: 169-219

Castañeda-Sánchez A. 2011. Propiedades nutricionales y antioxidantes del maíz azul (Zea mays L.). Revista temas selectos de ingeniería de alimentos 5-2:75-83

Castro, F. 2006. Colapsos ambientales-transiciones culturales. UNAM, BUAP. México

CEPAL, FAO, IICA. 2013. Perspectivas de la agricultura y del desarrollo rural en las

Revista Científica Biológico Agropecuaria Tuxpan 4 (1)

ISSN: 2007-6940 
Américas: Una mirada hacia América Latina y el Caribe 2014. Comisión Económica para América Latina y el Caribe (CEPAL), Organización de las Naciones Unidas para la Agricultura y la Alimentación (FAO), Instituto Interamericano de Cooperación para la Agricultura (IICA):220

Escobar, M.D. 2006. Valoración campesina de la diversidad del maíz. Estudio de caso de dos comunidades indígenas en Oaxaca, México. Tesis Doctoral, Universidad Autónoma de Barcelona, España.

Espinosa, T.E; Mendoza, C.M.D.C; Castillo, G.F; Ortiz, C.J y Delgado, A.A. 2010. Aptitud combinatoria del rendimiento de antocianinas y de características agronómicas en poblaciones nativas de maíz pigmentado. Revista fitotecnia mexicana 33(1):11-19 https://doi.org/10.35196/rfm.2010.1.11

FAO, 2016. Estadísticas sobre seguridad alimentaria. (Food and agriculture organization of the United Nations). [En línea], disponible en http://www.fao.org/economic/ess/ess-fs/es/ [Acceso el 14 de marzo 2016].

Fernández S. R; Morales, Ch.L.A y Gálvez, M.A. 2013. Importancia de los maíces nativos de México en la dieta nacional. Una revisión indispensable. Revista fitotecnia mexicana 36:275-283

https://doi.org/10.35196/rfm.2013.3-S3-A.275

Fitting, E. 2007. ¿La economía "natural" enfrenta a la global? Desafíos a los debates sobre el maíz. Revista bajo el volcán VII (11): 17-44.

Gil, A y Álvarez, N.M. 2007. El maíz criollo en la alimentación de las familias campesinas de Santiago Xalitzintla, Puebla. Colegio de Postgraduados Campus Puebla, Cholula Puebla. 23

Gobierno del estado de Tlaxcala. 1998. Monografías de los municipios de Tlaxcala.

González, U. 1995. El maíz y su conservación. Trillas, México. 399

Hernández, X.E. 1985. Agricultura tradicional y desarrollo en Xolocotzia obras de Efraín Hernández Xolocotzi. Revista de Geografía agrícola. Universidad Autónoma de Chapingo Tomo I. México: 419-422

Hernández-Vázquez M; Jiménez-García D; GarcíaJuárez G; Jiménez-López J; Orozco-Bolaños H; Hernández-Calva L.M y Morales-Acolzi T. 2015. Riesgo y vulnerabilidad del maíz de temporal en la región suroeste del estado de
Tlaxcala frente al cambio climático. Revista biológico agropecuaria tuxpan 3(5):10091018

INEGI. 2007. Anuario Estadístico Tomo II, Tlaxcala, México.

López, M.B; González, J.A; Díaz, S; Castro, I y García, Ll.M. 2007. Biodiversidad y bienestar humano: el papel de la diversidad funcional. Revista ecosistemas XVI (003): 68-79

Madrigal, A. 2008. Los campesinos se declaran inocentes: Cambios estructurales y contaminación genética del maíz criollo en san Antonio Atotonilco, Municipio de Ixtacuixtla, Tlaxcala, en Ortiz, Benjamín y Guy Duval (coordinadores). Sistemas complejos medio ambiente y desarrollo, Lupus Inquisitor, México: 97-132.

María, R. A; Salinas, M. Y y Taboada, G.O.R. 2003. Maíz azul de los valles altos de México. Rendimiento de grano y características agronómicas. Revista fitotecnia mexicana 25 (002): 101-107.

María, R.A; Hernández C.J.M; Muñoz, D L V. E y Ríos, S.A. 2015. Conocimiento de la diversidad y distribución actual del maíz nativo y sus parientes silvestres en México, segunda etapa 2008-2009. Instituto Nacional de Investigaciones Forestales, Agrícolas y Pecuarias. Centro de Investigación Regional Noroeste. Informe final Tlaxcala SNIBCONABIO, proyecto No. FZ016, México D.F

Massieu, Y y Lechuga, J. 2002. El maíz en México: Biodiversidad y cambios en el consumo. Revista análisis económico XVII (036): 281303

Meléndez, E.S.O. 2015. El K-C-P en las ciencias ambientales: un caso de estudio del maíz, los rituales y el ambiente de Ixtenco. Tesis de Licenciatura en Ciencias Ambientales, Universidad Autónoma de Tlaxcala, México.

Méndez-Montealvo, G; Solorza-Feria, J; Velázquez del Valle, M; Gómez-Montiel, N; ParedesLópez, O y Bello-Pérez, L.A. 2005. Composición química y caracterización calorimétrica de híbridos y variedades de maíz cultivadas en México. Revista agrociencia (39):267-274

Muñoz, A. 2003. Centli Maíz. Colegio de Postgraduados, Texcoco México. 210 
Núñez, I; González, G. E y Barahona A. 2003. La Biodiversidad: Historia y contexto de un concepto. Revista interciencia 28 (007): 387393.

Osorio-García, N; López-Sánchez, H; Gil-Muñoz, A; Ramírez-Valverde, B; Gutiérrez-Rangel, N; Crespo-Pichardo, G y Montero-Pineda, Á. 2012. Utilización, oferta y demanda de tecnología para producción de maíz en el valle de Puebla, México" en Revista agricultura, sociedad y desarrollo 9(1):55-69

Perales R.H; Brush, S.B y Qualset C.O. 2003. Landraces of maize in Central Mexico: an altitudinal transect. EconBot 57:7-20. https://doi.org/10.1663/0013-0001(2003)057[

Pérez, C.J.P; Y.I Beltrán, V y J.M Hernández, C. 2006. Avances en el rescate y conservación del maíz criollo en el estado de Hidalgo. Exploración etnobotánica e investigación agronómica. In: Investigación, Docencia y Patrimonio. Memorias de las Jornadas de Arqueología. Consejo Nacional para la Cultura y las Artes - Instituto Nacional de Antropología e Historia. México, D.F: 103113.

Rangel-Meza E; Muñoz-Orozco A; VázquezCarrillo G; Cuevas-Sánchez J; MerinoCastillo J y Miranda-Colín S. 2004. Nixtamalización, elaboración y calidad de tortilla de maíces de Acatlán Puebla, México. Revista agrociencia 38 (1): 53-61.

Reyes, G.G; Guerra, N.J y Calderón, P.G. 2005. Condiciones de cultivo del maíz criollo en comunidades de Puebla, Tlaxcala e Hidalgo: un análisis de las economías de autoconsumo. Revista aportes X (029): 63-82.

SAGARPA. 2007. Plan rector sistema producto maíz Tlaxcala, Tlaxcala.

Salinas, M.Y; Saavedra, A.S; Soria, R.J y Espinosa, T.E. 2008. Características fisicoquímicas y contenido de carotenoides en maíces (Zea mays L.) amarillos cultivados en el Estado de México. Revista agricultura técnica en México 34 (3): 357-364.

Santos, Ch.V.M; Zúñiga, E.M; Leos, R.J.A y Álvarez, M.A. 2014 Tipología de productores agropecuarios para la orientación de políticas públicas: Aproximación a partir de un estudio de caso en la región Texcoco, Estado de México, México. Revista sociedades rurales, producción y medio ambiente 14(28):47-69
Smale, M., y M. Bellon 2001. Economic Concepts for Designing Policies to Conserve Crop Genetic Resources on Farms. Rome, IPGRI.

Sarmiento, B y Castañeda, Y. 2011. Políticas públicas dirigidas a la preservación de variedades nativas de maíz en México ante la biotecnología agrícola. El caso del maíz cacahuancintle. Revista el cotidiano (166) 101-110.

Soleri, D; D. Cleveland y F. Aragón C. 2006. Transgenic Crops and Crop Varietal Diversity: The Case of Maize in Mexico. Journal BioScience 56 (6): 503-513. https://doi.org/10.1641/0006-3568(2006)56[5

Vázquez, C.M.G; Pérez, C.J.P; Hernández, C.J. M; Marrufo, D. M. L y Martínez, R.E. 2010. Calidad de grano y de tortillas de maíces criollos del altiplano y valle del mezquital, México. Revista fitotecnia mexicana 33 (4): 49-56.

Villa, M. 2008. ¿Qué hacemos con el campo mexicano? Mundi Prensa, México: 231. 
Copyright (c) 2016 Hermila Orozco Bolaños, Guillermina Garcia Juárez,

Maricela Hernández Vázquez y Maria G uadalupe Juárez Ortiz

(9) (1)

Este tex to está protegido por una licencia licencia CreativeCommons 4.0

Usted es libre para Compartir —copiar y redistribuir el material en cualquier medio o form ato—y Adaptar el documento —remezclar, transformar y crear a partir del material- para cualquier propósito,, incluso para fines comerciales, siempre que cumpla la condición de:

Atribución: Usted debe dar crédito a la obra original de manera adecuada, proporcionar un enlace a la licencia, e in dicar si se han realizado cam bios. Puede hacerlo en cualquier form a razonable, pero no de form a tal que sugiera que tiene el apoyo del licenciante o lo recibe por el uso que hace de la obra.

Resumenclelicencia - Textocompletodelalicencia 